\title{
Stochastic Channel-Based Federated Learning With Neural Network Pruning for Medical Data Privacy Preservation: Model Development and Experimental Validation
}

\author{
Rulin Shao ${ }^{1}, \mathrm{BA}$; Hongyu $\mathrm{He}^{2}$, BA; Ziwei Chen ${ }^{3}$, PhD; Hui Liu ${ }^{4}$, BA; Dianbo Liu ${ }^{5}$, PhD \\ ${ }^{1}$ Department of Mathematics and Statistics, Xi'an Jiaotong University, Xi'an, China \\ ${ }^{2}$ Department of Electrical Engineering, Xi'an Jiaotong University, Xi'an, China \\ ${ }^{3}$ Beijing Jiaotong University, Beijing, China \\ ${ }^{4}$ Department of Mathematics, Mianyang Vocational College, Mianyang, China \\ ${ }^{5}$ Computer Science and Artificial Intelligence Laboratory, Massachusetts Institute of Technology, Cambridge, MA, United States
}

Corresponding Author:

Dianbo Liu, PhD

Computer Science and Artificial Intelligence Laboratory

Massachusetts Institute of Technology

77 Massachusetts Ave

Cambridge, MA, 02139

United States

Phone: 16177101859

Email: dianbo@mit.edu

\section{Abstract}

Background: Artificial neural networks have achieved unprecedented success in the medical domain. This success depends on the availability of massive and representative datasets. However, data collection is often prevented by privacy concerns, and people want to take control over their sensitive information during both the training and using processes.

Objective: To address security and privacy issues, we propose a privacy-preserving method for the analysis of distributed medical data. The proposed method, termed stochastic channel-based federated learning (SCBFL), enables participants to train a high-performance model cooperatively and in a distributed manner without sharing their inputs.

Methods: We designed, implemented, and evaluated a channel-based update algorithm for a central server in a distributed system. The update algorithm will select the channels with regard to the most active features in a training loop, and then upload them as learned information from local datasets. A pruning process, which serves as a model accelerator, was further applied to the algorithm based on the validation set.

Results: We constructed a distributed system consisting of 5 clients and 1 server. Our trials showed that the SCBFL method can achieve an area under the receiver operating characteristic curve (AUC-ROC) of 0.9776 and an area under the precision-recall curve (AUC-PR) of 0.9695 with only $10 \%$ of channels shared with the server. Compared with the federated averaging algorithm, the proposed SCBFL method achieved a 0.05388 higher AUC-ROC and 0.09695 higher AUC-PR. In addition, our experiment showed that $57 \%$ of the time is saved by the pruning process with only a reduction of 0.0047 in AUC-ROC performance and a reduction of 0.0068 in AUC-PR performance.

Conclusions: In this experiment, our model demonstrated better performance and a higher saturating speed than the federated averaging method, which reveals all of the parameters of local models to the server. The saturation rate of performance could be promoted by introducing a pruning process and further improvement could be achieved by tuning the pruning rate.

(JMIR Form Res 2020;4(12):e17265) doi: 10.2196/17265

\section{KEYWORDS}

federated learning; differential privacy preserving; neural network pruning; health care; privacy; medical data; machine learning; neural network 


\section{Introduction}

\section{Medical Data Privacy}

Medical data analysis in health care brings many benefits and holds great promise for transforming the field. With the help of a wide range of health care networks, health care organizations are now able to analyze a vast volume of data with great variety and velocity to support decision making [1-3]. In addition, automated machine-learning algorithms could facilitate patients and physicians to make better informed choices by providing empirical estimates based on gigabytes of data [4]. Apart from decision support, medical data analysis could also promote analytical capability for patterns of use, analysis of unstructured data, predictive capability, and traceability [3].

However, health care data security and privacy issues have raised broad ethical and legal concerns in recent years given the sensitive nature of health information [5]. Health care research often involves studies of a large amount of data collected from various sources such as pharmacies, insurance companies, government agencies, and research institutions. For instance, to discover new drugs or assess a new therapy, a research institute may need clinical records provided by hospitals' autonomous databases [6]. This direct sharing of medical data is likely to violate individual privacy and expose data owners to the threat of illegal data collection [7].

To address this data privacy concern, different countries have enacted different legislations and policies [8,9], which impose limitations on data collection and utilization for health care research. Over the years, many traditional methods for privacy preserving have been proposed, such as deidentification [10,11], a hybrid execution model [12], and identity-based anonymization [13]. However, as pointed out by several authors, these methods alone could not guarantee the anonymity and security of medical data [14-16]. Recently developed machine-learning methods require considerable data to acquire models with sufficient accuracy [17]. To leverage massive and diverse datasets and promote machine-learning models, the issue of balancing privacy and regulatory requirements has to be addressed [18].

\section{Federated Learning}

In conventional deep learning, all training data are shared with a central server that performs the analysis. Having no control over this process, the clients that contribute the data may have to upload their sensitive information to the server without a guarantee of its security of privacy. Furthermore, the learned model is generally not directly available to the client so that they have to reveal the inputs to the cloud when using the model [19], risking privacy leakage in both the training and using processes. Federated learning can address this problem by introducing some algorithmic techniques that distribute the learning process to local devices so that the clients could keep their data private and obtain a local model for future use.

Federated optimization has been studied by Konečný et al $[20,21]$ for distributed optimization in machine learning. This work introduced a setting for distributed optimization in which none of the distinctive assumptions [21] is satisfied, making federated learning a feasible alternative to other methods. The proposed framework is different from conventional distributed machine learning [22-27] owing to the huge number of clients, extremely unbalanced/nonindependent and identically distributed data obtainable for each client, and poor network connections [28]. To address the latter constraint, Konečný et al [28] proposed two approaches to reduce the uplink communication costs: structured updates and sketched updates. McMahan et al [29,30] advocated for federated stochastic gradient descent (SGD) and federated averaging algorithms as feasible approaches for the federated learning of neural networks based on iterative model averaging. As an alternative to protecting a single data point's contribution to a learning a model [31], Geyer et al [32] proposed an algorithm for client-sided federated optimization to hide the specific contributions of individual clients during training. Further, methods to strengthen the reliability of federated learning, such as secure aggregation [33], essentially need synchronization on a rigid set of devices so that only a simple summation of the updates from users is consumed by the server side of the algorithm [34]. Applications based on federated learning algorithms have been proposed in several domains, ranging from content suggestions [35] to next-word prediction [36]. Bagdasaryan et al [37] focused on the vulnerability of federated learning. This work showed that the federated learning algorithm is vulnerable to a model-poisoning attack, which is different from poisoning attacks that target only the training data.

Besides the direct leakage of privacy mentioned above, participants in the distributed system may indirectly reveal some information about sensitive data via the weights uploaded to the server in the training process.

To address both direct and indirect privacy leakage of health care data, we developed the stochastic channel-based federated learning (SCBFL) method, which enables local participants to manipulate their data confidentially while benefitting the model's performance from the server with only a small proportion of the locally trained gradients revealed stochastically to the central model.

\section{Methods}

\section{Principle of the SCBFL Approach}

Based on the observation that different features do not contribute equally to the training process and that the importance of each feature may vary from one dataset to another, SCBFL (Figure 1) was developed as a privacy-preserving approach that seizes the most vital information from the local training results only by uploading a small fraction of gradients stochastically. The intuition behind this method is that the biological neural circuit follows the law of use and disuse, and the strongest neurons for an individual are those that constitute an active circuit in the learning process, suggesting that the neurons in one artificial neural network are not independent throughout a specific training process. Thus, we could consider the collaborative effect of neurons in each channel (similar to a biological neural circuit) when selecting parameters for a server update: if a channel of neurons changes substantially in a training loop, we can assume that it is a strong neural circuit in the network, 
corresponding to a sensitive feature in the input sets, whereas the neural channels showing little change in one training loop should be regarded as deteriorated channels, whose information could be kept private with minimal effect on the server's final performance. Choosing the channels with the most substantial variation enables SCBFL to only upload a small percentage of the gradients in each training loop while achieving comparable accuracy to the federated averaging method without uploading the integrate local weights to the server, as will be demonstrated in the Results section.

The update algorithm plays an essential role in SCBFL. In each global loop, SCBFL computes the norms of channels in gradients that result from the local training process, calculates the $\alpha$-percentile of the norms, and then sifts out the channels with greater variation in the gradients than the percentile, in which $\alpha$ is the update rate set by the local participant. The sifted parameters are then used for the server update.

Figure 1. Schematic of the stochastic channel-based federated learning (SCBF) model.

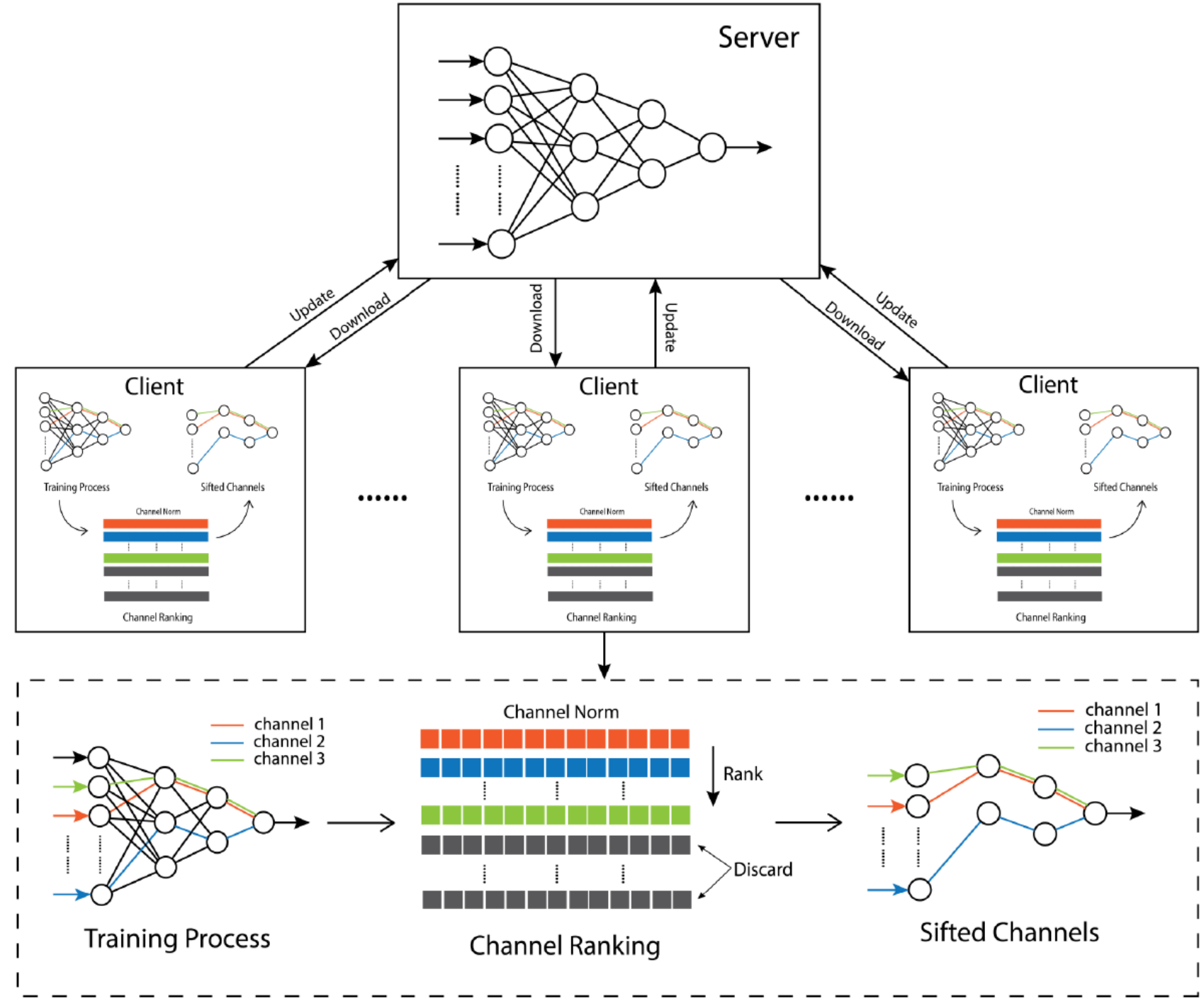

To facilitate the description of the algorithm, suppose there are $N$ features as input and an $L$-layer deep neural network is conducted with $m_{l}, m_{2}, \ldots, m_{L}$ neurons in each layer. For convenience, we denote $m_{0}=N$ as the input dimension, and we denote the weight matrix as $W=\left[W_{1}, W_{2}, \ldots W_{L}\right]$ and the bias matrix as $B=\left[B_{1}, B_{2}, \ldots B_{L}\right]$. The shapes of the weight matrix and bias matrix could be expressed as follows:

$$
W_{l}=\left(w_{l j}^{l}\right)_{m_{l-1} \times m_{i}}
$$

$$
B_{l}=\left(b_{i}^{l}\right)_{m_{i}}
$$

Where $l=1,2, \ldots L$, and $m_{l}$ is the number of neurons in the $l$ th layer.

The update algorithm includes five steps:

1. Train the local model: The local models are trained separately on their own datasets, and each model results in a gradient matrix showing the change in the weight matrix during each training loop. The gradient matrix $G$ has the same shape as the weight matrix $W$. Since the influence from the bias matrix is 
negligible compared to that of the weight matrix, the changes in bias are omitted for the sake of efficiency.

2. Compute channel norms: Considering that a channel must go through a neuron in each layer and correlates to an $L$-dimensional vector comprising the index of these neurons, the results of a channel's norm could be saved in an $L$-dimensional tensor $T$, each element of which equals a channel norm. The shape of $T$ should be:

$$
T=\left(t_{i_{2} i_{2} \cdots i_{L}}\right)_{i_{1} i_{2} \cdots i_{L}=1}^{m_{1} m_{2} \cdots-m_{L}}
$$

In addition, $c^{(i)}=\left[g_{0}^{(i)}, g_{1}^{(l)}, \cdots, g_{L}^{(i)}\right]$ is the $i$ th channel, in which $\vec{\imath}=\left[i_{1}, i_{2}, \cdots, i_{2}\right]$ is the index of the tensor that correlates the neurons this channel goes through in each layer. The Euclidean norm of each channel is calculated by

$$
n^{(l)}=\left|c^{(i)}\right|_{2}=\sum_{j=0}^{L}\left(g_{j}^{(i)}\right)^{2}
$$

and is saved in the $L$-dimensional tensor $T$ :

$$
T_{i_{1}, i_{2}, \cdots, i_{2}}=n^{(l)}=\left|c^{(l)}\right|_{2}=\sum_{j=0}^{L}\left(g_{j}^{(l)}\right)^{2} \text { (5) }
$$

3. Sort norms: Given a fixed upload rate $\alpha$ (also referred to as "update rate" in this paper), we could straighten the gradient tensor to a vector and sort it, computing the $\alpha$-quantile $q_{\alpha}$ as a threshold for the channel selection.
4. Process gradients: There are two ways to process the gradients. With negative selection, the channels with norms below the $\alpha$-quantile are discarded and the remaining parameters are selected for the update. With positive selection, the channels with a norm above $q_{\alpha}$ are selected and the remaining parameters are set to zeros.

In our preliminary trials, both selection methods worked well. Considering that different neural channels may include the same neurons, positive selection tends to behave better than negative selection due to the preference to upload more parameters with the same update rate. Taking positive selection as an example, for each element $T_{t 1},{ }_{t 2}, \ldots,{ }_{t L}$ in tensor $T$, which corresponds to a specific channel, the gradients are processed with respect to the rank of this channel's norm, as shown in the following form:

$$
\left(G_{i}\right)_{t_{l}}= \begin{cases}\left(G_{i}\right)_{t_{i}} & \text { if } T_{t_{1} t_{2} \cdots t_{L}}>q_{\alpha} \\ 0 & \text { else. }\end{cases}
$$

5. Update server: Finally, the processed gradient matrix $\mathrm{G}^{\sim}$ is uploaded to the server, and the server will update its parameters by adding gradients $\mathrm{G}^{\sim}$ to its original weights (Figure 2 ).

The server update algorithm is executed every global loop, and our experiment showed that with only $10 \%$ of local channels revealed, the server could have comparative performances to those of the federated averaging methods with higher speed to reach saturation. Before the next training loop begins, the local model downloads the server's latest weights. The download rate was set to $100 \%$ since we suppose that the server weights could be shared publicly, which could be adjusted according to the application scenarios.

Figure 2. Pseudocode of the server update.

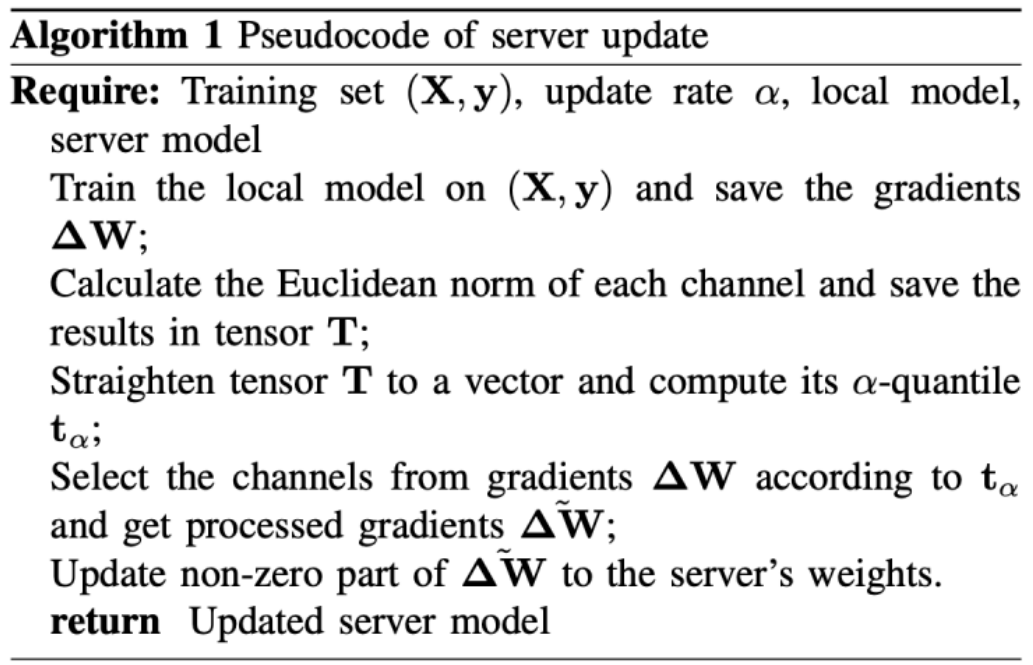

\section{Pruning Process}

Training a model with privacy-preserving methods could be time-consuming, especially when the training sets are enormous. To address this problem, we introduced a neural network pruning process to SCBFL that could prune off the redundant nodes in the neural network based on the validation set, thus saving a substantial amount of time. This work is done circularly in the first several global loops until the distributed system reaches a suitable scale, so that SCBFL with pruning (SCBFLwP) learns from the datasets more efficiently.

Neural network pruning (Figure 3) is not a novel concept. Yang [38] proposed a method to prune connections based on the magnitude of weights. He et al [39] used a channel-pruning method to accelerate a deep convolutional neural network. Han et al [40] introduced a growing-and-pruning approach for a fuzzy neural network. Moreover, Srinivas [41] proposed a systematic method to prune one neuron at a time, addressing 
the problem of pruning parameters in a trained neural network model.

Given the fact that each neural network has a computation process consisting of multiplication, addition, and activation, neurons whose output consists mostly of zeros may have little effect on the output of subsequent layers, not to mention the final results [31]. Removing these redundant nodes from the model will do little harm to the accuracy of the network but will save abundant execution time.

Average percentage of zeros (APoZ) [42], which measures the percentage of zeros in the activations of a neuron under rectified linear unit (ReLU) mapping, is used to evaluate the redundancy of neurons in the network. $\mathrm{CO}_{c}^{(i)}$ is denoted as the output of the $c$ th neuron in the $i$ th layer. Let $M$ denote the output dimension and $N$ denote the total quantity of validation examples. $A P_{O} Z_{c}^{(i)}$ of the $c$ th neuron in the $i$ th layer is then defined as:

$$
A P_{O} Z_{c}^{(l)}=A P_{O} Z\left(O_{c}^{(i)}\right)=\frac{\sum_{k}^{N} \sum_{j}^{M} f\left(o_{c_{r} j}^{(l)}(k)=0\right)}{N \times M}
$$

where $f(\cdot)=1$ if true and $f(\cdot)=0$ if false.

SCBFLwP (Figure 4) then decides which neurons will be pruned according to $\mathrm{APoZ}$ using validation sets: those having the highest APoZ will be pruned, the number of which is a fixed percentage of the total number of neurons left in each global loop.

Figure 3. Neural network pruning.

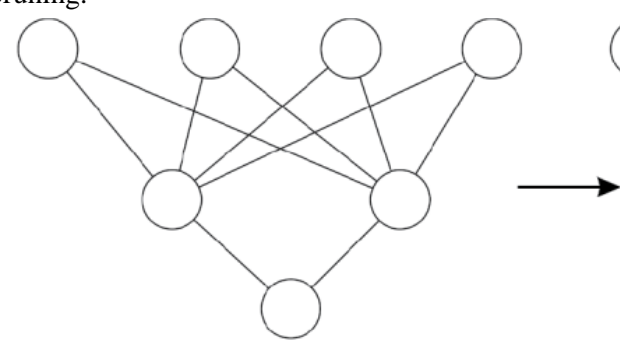

Before Pruning

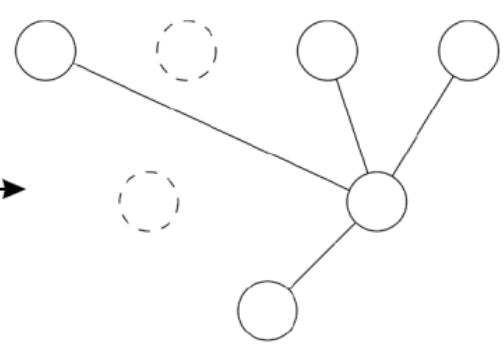

After Pruning

Figure 4. Pseudocode of stochastic channel-based federated learning with pruning (SCBFwP).

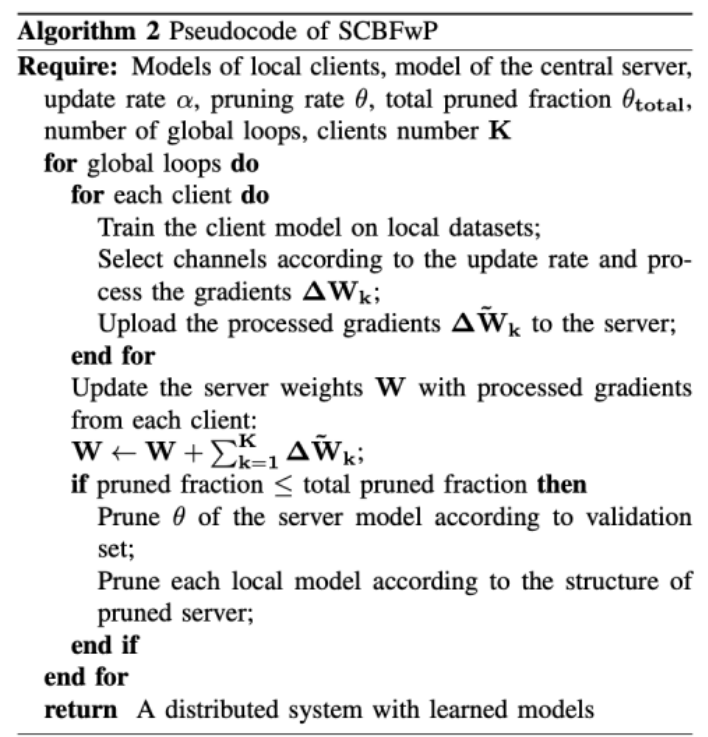

\section{Distributed Learning Setting}

We propose a privacy-preserving federated learning method based on the neural network. Federated learning could be executed on a distributed system such as a mobile device to achieve collaborative deep-learning goals with little risk of privacy leaks. Each device trains its model on the local dataset for several epochs in each global loop and only stochastically uploads a small percentage of the model weights to the server to achieve good performance in the server without sharing the local data or the overall model weights.
In our trial, we implemented a distributed system with 5 clients contributing to one server. Preliminary experiments were conducted to determine the proper structure for the proposed model. Through manual tuning, we found that the model achieves the best performance with high efficiency using 3 layers. Therefore, for each local client, we constructed an artificial neural network for binary prediction of mortality with 3 fully connected layers including 64, 32, and 1 neuron in the corresponding layers using ReLU activation at hidden layers and sigmoid activation at the output layer. We also added a dropout layer between the second and third hidden layers to reduce overfitting. 
Regarding the parameters of communication between server and clients, the download rate was set to $100 \%$ for each client model, supposing the parameters of the server are shared publicly. The update rate was set to $30 \%$ for both the channel-based federated learning method and distributed selective SGD method. To enhance the influence of the latest update parameters, we chose 0.8 as the decay rate. As for the training process, we trained each model for 100 global loops and 5 epochs in each loop with the batch size set to 32 .

We used the SGD algorithm to optimize our neural networks. Concerning the configuration of SGD, the learning rate is a hyperparameter that controls how much to adjust the model in response to the estimated error each time when the model weights are updated. Our experiments on testing models with various learning rates suggested that the proper learning rate was around 0.01 to guarantee both good performance and stable results.

In addition to the configuration of the model, the importance of performance measurement has long been recognized. With respect to the assessment of a classification model, area under the receiver operating characteristic curve (AUC-ROC) and area under the precision recall curve (AUC-PR) are reliable metrics: the higher the AUC value, the better the model is at distinguishing between patients in terms of mortality and survival.

\section{Dataset for the Experiment}

The data used in our experiment were provided by hospitals, comprising 30,760 admissions with status information represented by alive or dead. To explore the relationship between mortality and admissions, we developed a model that takes the medications as inputs and predictions of binary mortality as the output. The cohort was managed for 2917 different medications in total. Information on whether a patient took each of the medications after admission was adopted as a binary input feature. We used $60 \%$ of the dataset for training, $10 \%$ as the validation set, and $30 \%$ as the test set. The training set was equally divided into five parts as local training sets.

\section{Statistical Analysis}

The performances of models were evaluated by the AUC-ROC and AUC-PR, which are both typically used for measuring the performance of a classifier. To better understand the ROC curve, the concept of a confusion matrix first needs to be introduced.

A confusion matrix is a table consisting of four different combinations of prediction and ground truth, which are the true positive (TP), false positive (FP), false negative (FN), and true negative (TN). TP means that the model predicts the sample as positive and it is in fact positive. The value of TP can be calculated by counting the number of correct positive predictions. The other three parameters can be interpreted in a similar manner. With the help of a confusion matrix, more performance indicators can be defined, including the true positive rate (TPR), also known as recall and sensitivity, the false positive rate (FPR), and precision, which are calculated as follows:

\section{$\mathrm{TPR}=\mathrm{TP} / \mathrm{TP}+\mathrm{FN}(\mathbf{8})$}

$\mathrm{FPR}=\mathrm{FP} / \mathrm{TN}+\mathrm{FP}(\mathbf{9})$

\section{Precision=TP/TP+FP (10)}

The ROC curve is plotted with TPR against FPR at various classification thresholds, where TPR is on the y-axis and FPR is on the $\mathrm{X}$-axis. Lowering the classification threshold means that the model will predict more samples as positive, thus increasing both FPR and TPR. As an alternative to ROC, the PR curve is plotted with precision against recall (TPR) at various classification thresholds. When datasets are imbalanced or skewed, the PR curve is a preferred alternative to the ROC curve. Both curves provide a visualization of model performance at different thresholds, and the AUC measures the entire two-dimensional area under these curves, providing an aggregate measurement of performance across all possible thresholds. Ranging in value from 0 to 1, AUC-ROC and AUC-PR can be interpreted as the possibility that the model ranks a positive sample more highly than a negative sample.

\section{Results}

\section{Performance of the SCBFL Model}

The update rate controls the number of selected channels whose nonzero part is uploaded to the server in each global loop, playing a vital role in affecting the final performance. To choose a suitable update rate for our distributed system, we implemented SCBFL models with different update rates ranging from $10 \%$ to $100 \%$. Neural network pruning was used in this step to accelerate the training process. The performances are plotted in the first row of Figure 5. The result showed that even with $10 \%$ of the channels uploaded to the server, the SCBFL model achieved an AUC-ROC of 0.9776 and an AUC-PR of 0.9695 , which outperformed the model that shared all of the parameters with the server. In addition, using a wide range of upload rates only led to a 0.01319 amplitude change in AUC-ROC and a 0.02739 amplitude change in AUC-PR, which facilitated the configuration process with stably high performance. 
Figure 5. Performances of stochastic channel-based federated learning (SCBF) models. The top two graphs show the performances of SCBF with pruning (SCBFwP) using different update rates, and the bottom two graphs show the comparison between the performances of SCBF and federated averaging (FA). The left column shows the area under the receiving operating characteristic curve (AUC-ROC) and the right column shows the area under the precision-recall curve (AUC-PR) as performance metrics.
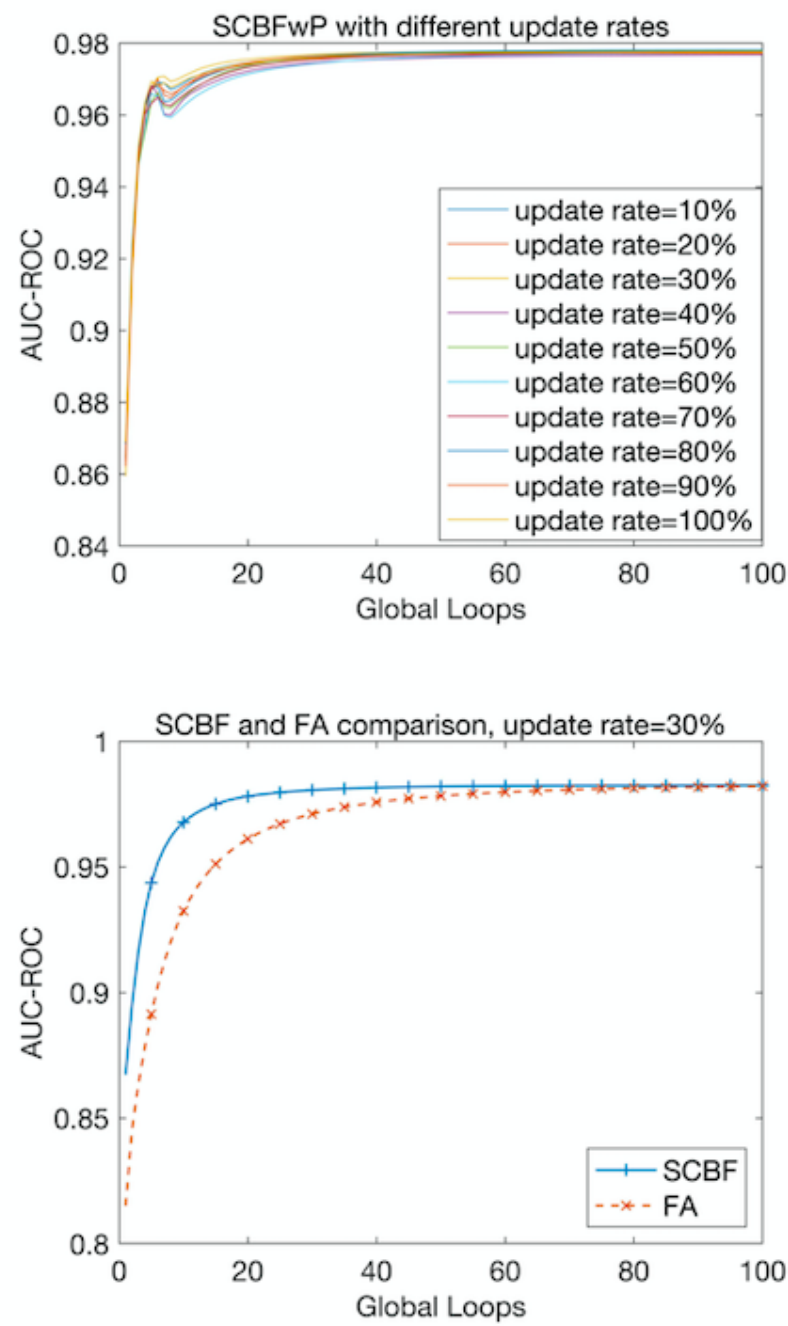

Table 1 compares the effectiveness of the SCBFL method with that of federated averaging, which is widely used in distributed systems and implements the federated learning by averaging the gradients obtained from local training processes [36]. For this comparison, we set the update rate to $30 \%$ for SCBFL and conducted both methods for federated learning on the same datasets for 100 global loops without pruning. As shown in Figure 5, our model reached saturation at the 20th global loop,
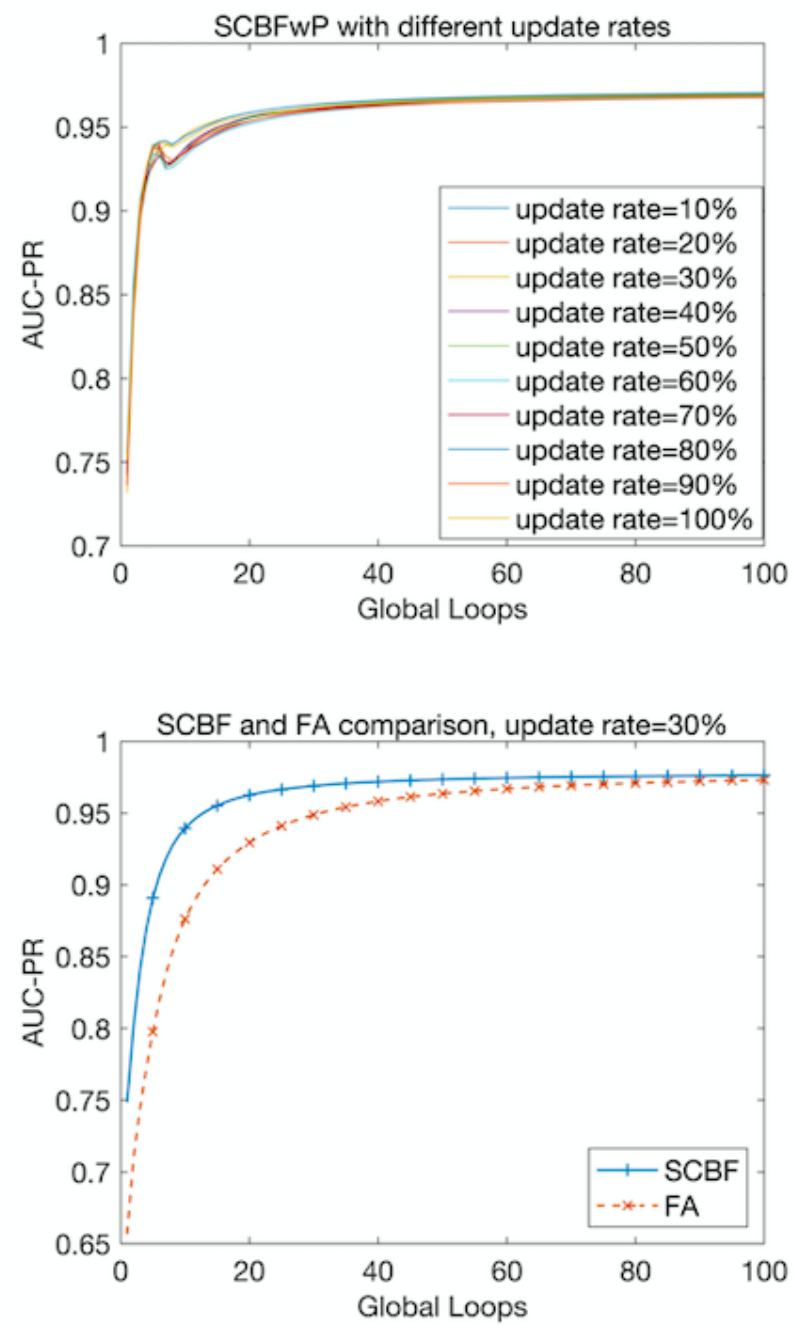

which was faster than that obtained with federated average, which reached saturation at the 60th global loop. The performance of SCBFL consistently exceeded that of federal averaging. In the 4th global loop, SCBFL achieved a 0.05388 higher AUC-ROC and 0.09695 higher AUC-PR than those of federated averaging. After 100 global loops, the AUC-ROC and AUC-PR of SCBFL was 0.0033 and 0.0032 higher than that of federated averaging, respectively.

Table 1. Saturated performances of stochastic channel-based federated learning compared with federated averaging.

\begin{tabular}{lll}
\hline Method & AUC-ROC $^{\mathrm{a}}$ & AUC-PR $^{\mathrm{b}}$ \\
\hline SCBFL $^{\mathrm{c}}$ & 0.9825 & 0.9763 \\
Federated averaging & 0.9821 & 0.9731 \\
\hline
\end{tabular}

a AUC-ROC: area under the receiver operating characteristic curve.

${ }^{\mathrm{b}} \mathrm{AUC}-\mathrm{PR}$ : area under the precision-recall curve.

${ }^{\mathrm{c}} \mathrm{SCBFL}$ : stochastic channel-based federated learning.

As shown in Figure 6, when the upload rate for the channels was set to $30 \%, 45 \%$ of the parameters were uploaded to the server using positive selection. With half of the parameters unrevealed to the server, the model achieved better performance and higher saturating speed. 
Figure 6. Trans-information for upload processes using different methods. The stochastic channel-based federated learning with pruning (SCBFwP) method could save $85 \%$ of the trans-information compared to federated averaging (FA), and stochastic channel-based federated learning (SCBF) could save $55 \%$ compared with FA.

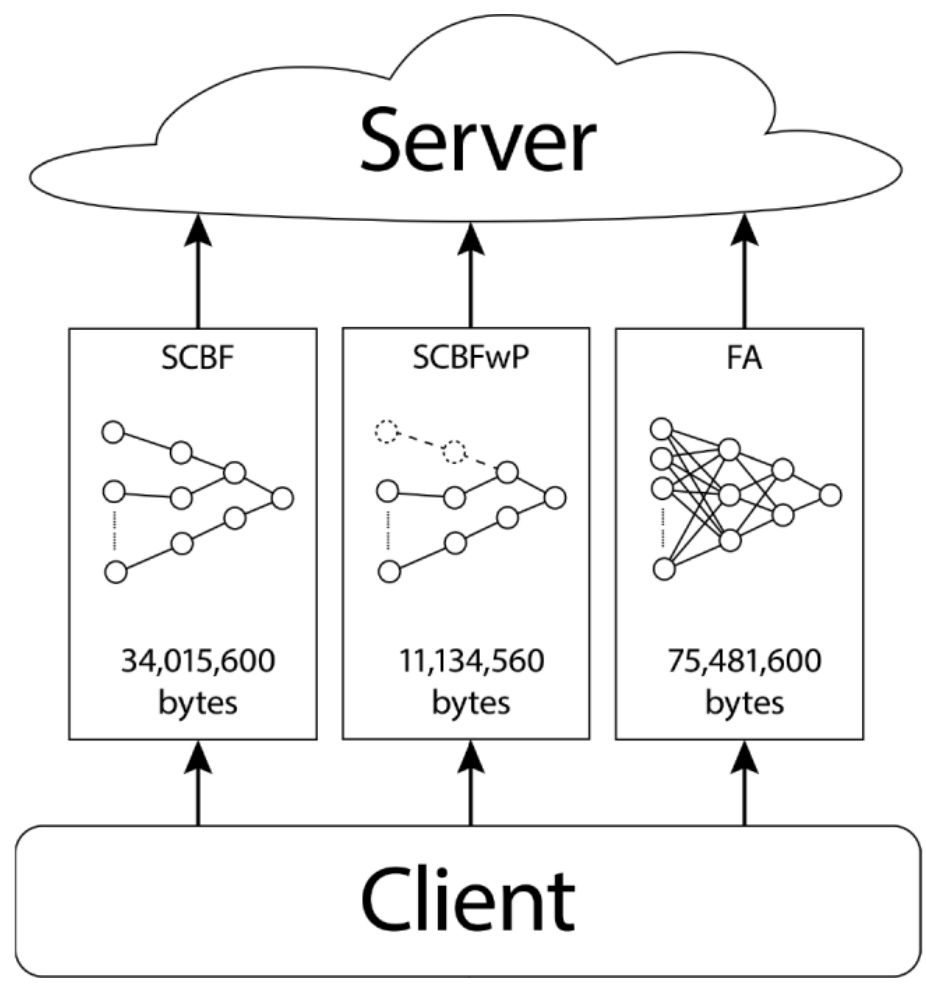

\section{Performance of SCBFLwP}

To speed up the training process and reduce the size of the neural network, we conducted network pruning for several loops after pretraining the model. In our trials, we set the pruning rate for each global loop to $10 \%$, which represents the proportion of neurons to be pruned in the training loop. The total proportion of neurons to be pruned in the first several loops was set to $47 \%$, which determines the final scale of the pruned model. Table 2 summarizes the performance of the SCBFLwP method for different update rates. Figure 7 compares the AUC-ROC and AUC-PR values of the SCBFL and federated averaging models with and without pruning.

Table 2. Saturated performances of stochastic channel-based federated learning with pruning with different update rates.

\begin{tabular}{lll}
\hline Update rate & AUC-ROC $^{\mathrm{a}}$ & AUC-PR $^{\mathrm{b}}$ \\
\hline $10 \%$ & 0.9776 & 0.9695 \\
$20 \%$ & 0.9772 & 0.9686 \\
$30 \%$ & 0.9777 & 0.9697 \\
$40 \%$ & 0.9768 & 0.9604 \\
$50 \%$ & 0.9780 & 0.9695 \\
$60 \%$ & 0.9774 & 0.9682 \\
$70 \%$ & 0.9774 & 0.9688 \\
$80 \%$ & 0.9781 & 0.9703 \\
$90 \%$ & 0.9774 & 0.9676 \\
$100 \%$ & 0.9775 & 0.9685 \\
\hline
\end{tabular}

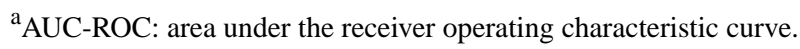

${ }^{b}$ AUC-PR: area under the precision-recall curve. 
Figure 7. Performance of stochastic channel-based federated learning with pruning (SCBFwP). The top two graphs show the comparison between stochastic channel-based federated learning (SCBF) and federating averaging (FA) with and without pruning. The middle graphs show the performance of SCBFwP with different pruning rates. The performances of SCBFwP with different numbers of total pruned neurons are shown in the bottom two graphs. The left column shows the area under the receiver operating characteristic curve (AUC-ROC) and the right column shows the area under the precision-recall curve (AUC-PR) as performance metrics.
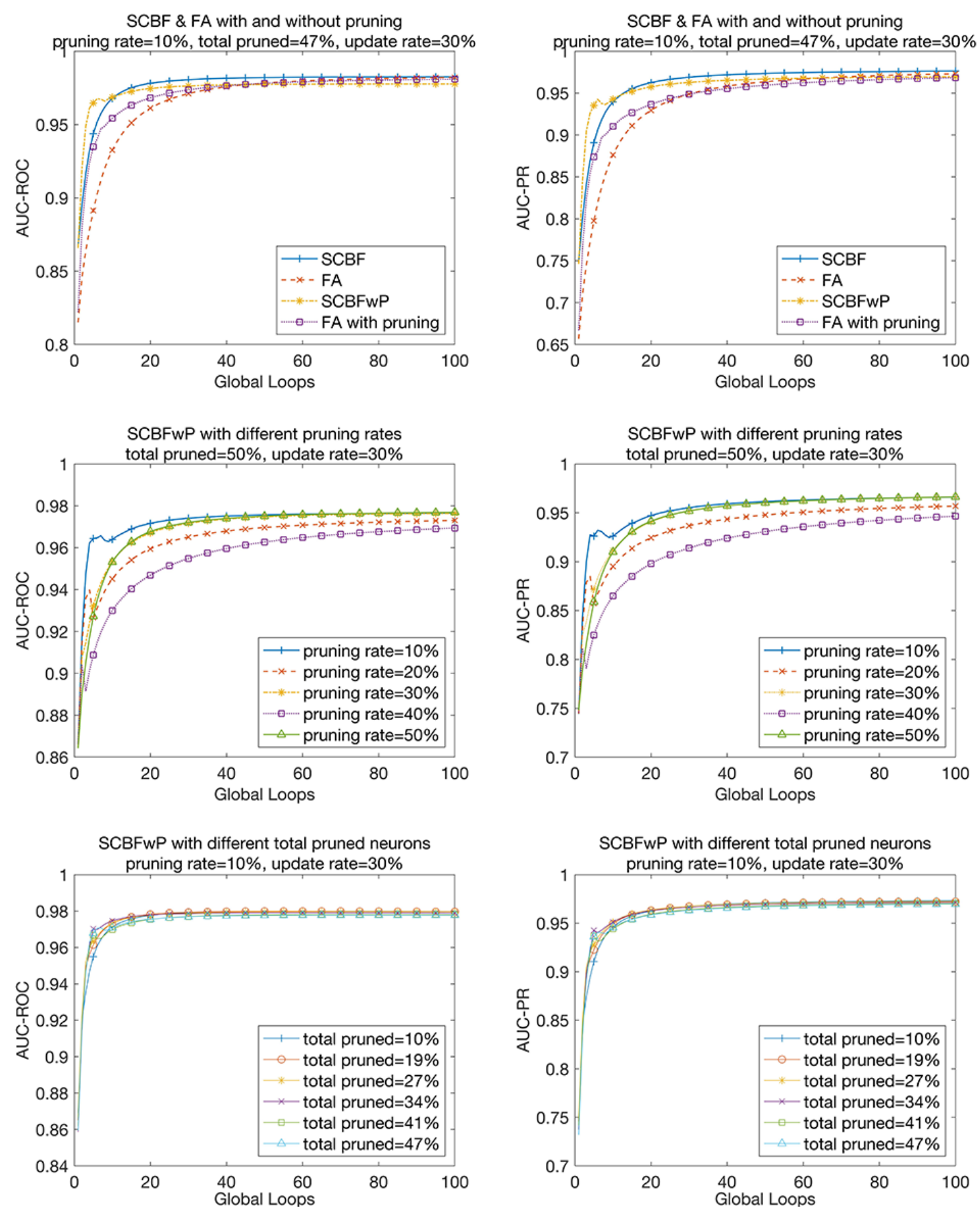

As shown in Table 3, the AUC-ROC for SCBFL with pruning was reduced by 0.0048 and the AUC-PR was reduced by 0.006814 . There was a reduction of 0.0012 in AUC-ROC and

of 0.0047 in AUC-PR for the federated averaging method compared to federated averaging with pruning. 
Table 3. Saturated performances of stochastic channel-based federated learning (SCBFL) and federated averaging (FA) with and without pruning.

\begin{tabular}{lll}
\hline Methods & AUC-ROC $^{\mathrm{a}}$ & AUC-PR $^{\mathrm{b}}$ \\
\hline FA & 0.9821 & 0.9731 \\
SCBFL & 0.9825 & 0.9763 \\
FAwP $^{\mathrm{c}}$ & 0.9809 & 0.9683 \\
SCBFLwP $^{\mathrm{d}}$ & 0.9776 & 0.9694 \\
\hline
\end{tabular}

${ }^{\mathrm{a}} \mathrm{AUC}-\mathrm{ROC}$ : area under the receiver operating characteristic curve.

${ }^{\mathrm{b}} \mathrm{AUC}-\mathrm{PR}$ : area under the precision-recall curve.

${ }^{\mathrm{c}}$ FAwP: federating averaging with pruning.

${ }^{\mathrm{d}}$ SCBFLwP: stochastic channel-based federated learning with pruning.

Moreover, the best performance was achieved by SCBFL after 100 loops of training with an AUC-ROC of 0.9825 and an AUC-PR of 0.9763 (Table 3). The highest evaluation in the first 5 loops was obtained by the SCBFL model with pruning.

To assess the stability of our model with the pruning rate and total pruned fraction (also called the total pruned rate), we executed the models of SCBFLwP controlling the variate. First, we fixed the total pruned fraction to $50 \%$ and ran the programs with different pruning rates ranging from $10 \%$ to $50 \%$ (Table
4). As shown in Figure 7, with an increase in the pruning rate, the final performance improved and saturated more quickly under most circumstances. However, there were also exceptions with higher performances at a $10 \%$ pruning rate for both AUC-ROC and AUC-PR, and lower performances at a $40 \%$ pruning rate for AUC-PR. In the graphs in the bottom row of Figure 7, we fixed the pruning rate to $10 \%$ and executed pruning for different times ranging from 1 to 6 (Table 5). The total pruned fractions were calculated and are annotated in the corresponding labels.

Table 4. Saturated performances of stochastic channel-based federated learning with pruning when the total pruned proportion was fixed and the pruning rate for each training loop changed.

\begin{tabular}{lll}
\hline Pruning rate/loop & AUC-ROC $^{\mathrm{a}}$ & AUC-PR \\
\hline $10 \%$ & 0.9765 & 0.9661 \\
$20 \%$ & 0.9730 & 0.9568 \\
$30 \%$ & 0.9763 & 0.9662 \\
$40 \%$ & 0.9693 & 0.9465 \\
$50 \%$ & 0.9769 & 0.9663 \\
\hline
\end{tabular}

${ }^{\mathrm{a}} \mathrm{AUC}-\mathrm{ROC}$ : area under the receiver operating characteristic curve.

${ }^{\mathrm{b}} \mathrm{AUC}-\mathrm{PR}$ : area under the precision-recall curve.

Table 5. Saturated performances of stochastic channel-based federated learning with pruning when the pruning rate for each training loop was fixed and the total pruned proportion changed.

\begin{tabular}{lll}
\hline Total pruned proportion & AUC-ROC $^{\mathrm{a}}$ & AUC-PR \\
\hline $10 \%$ & 0.9769 & 0.9731 \\
$19 \%$ & 0.9797 & 0.9722 \\
$27 \%$ & 0.9795 & 0.9725 \\
$34 \%$ & 0.9789 & 0.9714 \\
$41 \%$ & 0.9781 & 0.9703 \\
$47 \%$ & 0.9778 & 0.9697 \\
\hline
\end{tabular}

${ }^{\mathrm{a}} \mathrm{AUC}-\mathrm{ROC}$ : area under the receiver operating characteristic curve.

${ }^{b}$ AUC-PR: area under the precision-recall curve.

As shown in Figure 6, the SCBFLwP could save 85\% of the trans-information compared to federated averaging. For SCBFL, when the upload rate for channels was set to $30 \%, 45 \%$ of the parameters were uploaded to the server using positive selection.

\section{Running Time}

SCBFL preserves the privacy of data by adding a channel-based upload algorithm, which will lead to an increased burden of calculations when applied to a complex neural network. 
However, this problem could be addressed by introducing a pruning process in several global loops. To illustrate this, the times consumed by SCBFL and federated averaging before and after pruning described in the last section are compared in Table 6 . The pruning process could reduce $57 \%$ of the time for SCBFL and $48 \%$ of the time consumed by federated averaging.

Table 6. Time consumed by stochastic channel-based federated learning (SCBFL) and federated averaging with and without pruning.

\begin{tabular}{ll}
\hline Methods & Time (seconds) \\
\hline Federated averaging & 8679 \\
Federated averaging with pruning & 4508 \\
SCBFL & 19,696 \\
SCBFL with pruning & 8469 \\
\hline
\end{tabular}

Table 7 shows that models with lower update rates tended to that choosing a lower rate for the update could better preserve consume less time than those with larger update rates, indicating the privacy as well as save time.

Table 7. Time consumed by stochastic channel-based federated learning with pruning with different update rates.

\begin{tabular}{ll}
\hline Update rate & Time (seconds) \\
\hline $10 \%$ & 8339 \\
$20 \%$ & 8545 \\
$30 \%$ & 8469 \\
$40 \%$ & 7987 \\
$50 \%$ & 8359 \\
$60 \%$ & 12,577 \\
$70 \%$ & 9278 \\
$80 \%$ & 11,462 \\
$90 \%$ & 13,169 \\
$100 \%$ & 13,030 \\
\hline
\end{tabular}

Table 8 shows that different pruning rates for each global loop executing time of the pruning process. With a fixed pruning can equally save time. In addition, the model will consume more time if the number of pruned neurons is too small due to the rate, the time consumed by the model tended to decrease by reducing the model size.

Table 8. Time consumed by stochastic channel-based federated learning with pruning using different pruning rates for each loop or different total pruned proportions.

\begin{tabular}{cl}
\hline Pruning parameter & Time (seconds) \\
\hline Pruning rate/loop & 11,144 \\
$10 \%$ & 8561 \\
$20 \%$ & 11,852 \\
$30 \%$ & 8389 \\
$40 \%$ & 12,000 \\
$50 \%$ & \\
Total pruned & 25,755 \\
$10 \%$ & 22,717 \\
$19 \%$ & 17,579 \\
$27 \%$ & 15,909 \\
$34 \%$ & 8050 \\
$41 \%$ & 8469 \\
$47 \%$ & \\
\hline
\end{tabular}




\section{Discussion}

\section{Principal Results}

The proposed SCBFL method computes the norms of channels in gradients resulting from the local training output after each global loop, calculates the $\alpha$-percentile of the channel norms, and then sifts out the channels that have greater variation in the gradients compared with the percentile for the server update. In this method, the server seizes the information from the uploading channels with the biggest variation, achieving comparative performance to the state-of-the-art method (federated averaging), which has to convey the entire local weights to the server when updating. Figure 1 shows the relationship between the server and clients, and demonstrates the process of the server update. This confirms the intuition behind SCBFL: the importance of a feature differs when training on different datasets, and thus important information can be extracted from the channels through which features with the greatest variation pass. We could infer that less than $10 \%$ of the channels contain the most fundamental information and that ignoring the remaining information does little harm to the learning of models.

It is important to train a small-scaled deep-learning model with high processing speed. The results showed that network pruning could speed up the training process and accelerate convergence while maintaining higher performance. As expected, pruning $47 \%$ of the neurons from the network decreased the final performance due to the simplified model structure. The reduction in performance is negligible in many application situations but the acceleration in both saturating and training speed is quite beneficial, as discussed further below. Overall, these results demonstrate that SCBFL is a reliable choice for federated learning, and that the SCBFLwP method might be a better choice when a quicker saturating speed is desired.

The graphs in the first row of Figure 7 show an obvious decline in the performance of SCBFLwP, which indicates an overpruned phenomenon for our trials. This indicates a tradeoff between time efficiency and the final accuracy. However, by tuning the pruning rate for each global loop and the total pruned rate of the model, we could achieve better performance. This is because if only the redundant neurons are pruned, the model could promote its learning efficiency without retaining useless information.

Figure 7 also shows that the performance of SCBFL improved when the times of pruning were reduced. The results with a fixed pruning rate were more stable than those with a fixed total pruned rate, indicating that more attention should be paid to the selection of the pruning rate for each step when building models, and it is stable for a SCBFL model to adjust the times of neural network pruning. Therefore, after choosing a suitable pruning rate, we could appropriately increase the loops in which the model was pruned to shorten the execution time with little effect on the final performance.

\section{Differential Privacy Preservation}

Differential privacy [23,43-46], as a strong criterion for privacy-preserving, is defined when the probability of a given output does not primarily depend on the involvement of a data point in the inputs [19]. This is useful because conventional deep learning has raised substantial privacy concerns, which may prevent a company from collecting data for model training. A model-inversion attack may extract parts of the training data through a deep-learning network, as demonstrated by Fredrikson et al [47]. One might attempt to reduce the risk of privacy leakage by adding noise to the parameters that result from the training process. However, it is hard to achieve a balance between performance and privacy preservation since stronger noise offers protection for privacy as well as worse performance. Therefore, we have been seeking methods that can help to preserve local privacy during the training process.

To address this issue, the SCBFL method realizes the function of differential privacy preservation by protecting the two sources of potential privacy leakages from federated learning: the actual values of uploaded gradients from the local participants and the mechanism by which these gradients are chosen [19]. By setting a threshold to select the parameters of gradients channel-wise, the actual values uploaded to the server are stored in a sparse tensor that is processed from SGD, a stochastic training process that has already been used for many privacy-preserving cases $[48,49]$. In addition, the participant could independently choose the update rate for their models, thus making it hard to track the selection of the channels used for the update, especially when they are trained individually using different datasets.

\section{Limitations}

We proposed an update algorithm that plays a vital role in SCBFL. This algorithm involves calculation of the $\alpha$-percentile of the norms and searching for channels with greater variation in the gradients than the percentile. Given the massive size of input features, the model structure has to be extended to reach high performance. However, as is the case for most deep-learning models with complex structures, the time complexity will increase with the expansion of model size. Although the neural network pruning method has been introduced to reduce the executing time, the performance of the model will slightly decrease because of the simplified model structure. Moreover, differential privacy could be further conducted with our models to evaluate the privacy-preserving ability quantitatively.

\section{Comparison With Prior Work}

A large and representative dataset is usually required to train a neural network model. The dataset may contain sensitive information and the models should not expose the private information. Conventional methods that rely on a centrally trained model have a higher risk of privacy leakage. In conventional deep learning, the owners of the data cannot control the learning objective and have no idea of what can be inferred from their data. The federated averaging method represented progress in this regard by using iterative model averaging. Nevertheless, this approach still involves the exposure of all model parameters. The proposed SCBFL method, which improves server performance by uploading only a proportion of gradients, could address both direct and indirect privacy leakage concerns. In addition, an inverse-model attack, which extracts information from the uploaded parameters, could 
be hindered by the stochastic nature of our upload algorithm taking advantage of SGD. We found that even with only $10 \%$ of the channels uploaded to the server, the SCBFL model achieved an AUC-ROC of 0.9776 and an AUC-PR of 0.9695, outperforming the models that share all parameters with the server. As shown in our trials, after 100 global loops, the AUC-ROC and AUC-PR of SCBFL was 0.0033 and 0.0032 higher than that of federated averaging, respectively. Therefore, we could conclude that our method achieves comparative performance to the federated averaging method but with a higher saturating speed.

\section{Conclusions}

We proposed a privacy-preserving approach for distributed systems whose models are trained based on any type of neural network. Our methodology involved development of a channel-based update algorithm for the server, enabling the system to achieve state-of-the-art performance without forcing the participants to reveal their inputs or model weights to the server. Addressing both direct and indirect privacy leakage concerns, our model uploads a fraction of channels in the gradients from local models to the server and could achieve better performance with only $10 \%$ of the channels uploaded, thereby reducing the redundancy of gradients while preserving privacy. Inverse-model attack, which analyzes information from the uploaded parameters, could be obstructed by the stochastic nature of our upload algorithm taking advantage of SGD. Moreover, we introduced a neural pruning process to the model, which could accelerate the training process and saturating speed with little sacrifice to the final performance. Experimental validation showed that neural network pruning could efficiently speed up the training process as well as the saturation of performance. Moreover, better performance was achieved when tuning the pruning proportion to cut off the redundant neurons in several training loops.

\section{Acknowledgments}

ZC received financial support from the "Fundamental Research Funds for the Central Universities" (2019RC032). The authors would like to thank the Department of Biomedical Informatics of Harvard Medical School for provision of the dataset.

\section{Authors' Contributions}

RS wrote and revised the manuscript, conceived of the method, implemented the models, and conducted the methodological analysis. DL conceived of the methodology, provided scientific oversight, and revised the manuscript. HH performed the experiments, and wrote and revised the manuscript. HL performed the experiments and revised the manuscript.

\section{Conflicts of Interest}

None declared.

\section{References}

1. Watson HJ. Tutorial: Big Data Analytics: Concepts, Technologies, and Applications. Commun Assoc Inf Syst $2014 ; 34$. [doi: $10.17705 / 1$ cais.03465]

2. Raghupathi W, Raghupathi V. Big data analytics in healthcare: promise and potential. Health Inf Sci Syst 2014;2:3 [FREE Full text] [doi: 10.1186/2047-2501-2-3] [Medline: 25825667]

3. Wang Y, Kung L, Byrd TA. Big data analytics: Understanding its capabilities and potential benefits for healthcare organizations. Technol Forecast Soc Change 2018 Jan;126:3-13. [doi: 10.1016/j.techfore.2015.12.019]

4. Peterson ED. Machine Learning, Predictive Analytics, and Clinical Practice: Can the Past Inform the Present? JAMA 2019 Nov 22. [doi: 10.1001/jama.2019.17831] [Medline: 31755902]

5. Adam N, White T, Shafiq B, Vaidya J, He X. Privacy preserving integration of health care data. AMIA Annu Symp Proc 2007 Oct 11:1-5 [FREE Full text] [Medline: 18693786]

6. Bertino E, Ooi B, Yang Y, Deng R. Privacy and ownership preserving of outsourced medical data. : IEEE; 2005 Apr 18 Presented at: 21st International Conference on Data Engineering (ICDE'05); 2005; Tokoyo, Japan p. 521-532. [doi: 10.1109/ICDE.2005.111]

7. Abouelmehdi K, Beni-Hessane A, Khaloufi H. Big healthcare data: preserving security and privacy. J Big Data 2018 Jan 9;5(1):1. [doi: 10.1186/s40537-017-0110-7]

8. Craig T, Ludloff M. Privacy and big data: the players, regulators, and stakeholders. Sebastopol, California: O'Reilly Media, Inc; Sep 2011.

9. Jensen M. Challenges of Privacy Protection in Big Data Analytics. : IEEE; 2013 Sep 16 Presented at: 2013 IEEE International Congress on Big Data; June 27-July 2, 2013; Santa Clara, CA p. 235-238. [doi: 10.1109/bigdata.congress.2013.39]

10. Li N, Li T, Venkatasubramanian S. t-Closeness: Privacy Beyond k-Anonymity and l-Diversity. : IEEE; 2007 Jun 4 Presented at: 2007 IEEE 23rd International Conference on Data Engineering; April 15-20, 2007; Istanbul, Turkey p. 106-115. [doi: 10.1109/icde.2007.367856]

11. Machanavajjhala A, Gehrke J, Kifer D, Venkitasubramaniam M. L-diversity: privacy beyond k-anonymity. : IEEE; 2006 Apr 24 Presented at: 22nd International Conference on Data Engineering (ICDE'06); April 3-7, 2006; Atlanta, GA p. 24. [doi: 10.1109/ICDE.2006.1] 
12. Jain P, Gyanchandani M, Khare N. Big data privacy: a technological perspective and review. J Big Data 2016 Nov 26;3(1):25. [doi: $10.1186 / \mathrm{s} 40537-016-0059-\mathrm{y}$ ]

13. Sedayao J, Bhardwaj R, Gorade N. Making Big Data, Privacy, and Anonymization Work Together in the Enterprise: Experiences and Issues. : IEEE; 2014 Sep 25 Presented at: 2014 IEEE International Congress on Big Data; June 27-July 2, 2014; Anchorage, AK p. 601-607. [doi: 10.1109/bigdata.congress.2014.92]

14. Hundepool A, Willenborg L. Chapter 5: $\mu$ - and $\tau$-ARGUS: Software for Statistical Disclosure Control. In: Record Linkage Techniques 1997: Proceedings of an International Workshop and Exposition. Washington, DC: National Academies Press; 1997.

15. Samarati P. Protecting respondents identities in microdata release. IEEE Trans Knowl Data Eng 2001 Dec;13(6):1010-1027. [doi: 10.1109/69.971193]

16. Sweeney L. In: Lin TY, Qian S, editors. Datafly: a system for providing anonymity in medical data. Boston, MA: Springer; 1998:356-381.

17. Mohassel P, Zhang Y. SecureML: A System for Scalable Privacy-Preserving Machine Learning. : IEEE; 2017 Jun 26 Presented at: 2017 IEEE Symposium on Security and Privacy (SP); May 22-26, 2017; San Jose, CA p. 17-38. [doi: 10.1109/sp.2017.12]

18. Rajkomar A, Dean J, Kohane I. Machine Learning in Medicine. N Engl J Med 2019 Apr 04;380(14):1347-1358. [doi: 10.1056/NEJMra1814259] [Medline: 30943338]

19. Shokri R, Shmatikov V. Privacy-Preserving Deep Learning. : Association for Computing Machinery; 2015 Presented at: Proceedings of the 22nd ACM SIGSAC Conference on Computer and Communications Security; October 2015; New York, NY p. 1310-1321. [doi: 10.1145/2810103.2813687]

20. Konečný J, McMahan B, Ramage D. Federated Optimization:Distributed Optimization Beyond the Datacenter. arXiv. 2015 Nov 11. URL: http://arxiv.org/abs/1511.03575 [accessed 2019-11-15] [WebCite Cache ID https://arxiv.org/abs/1511.03575]

21. Konečný J, McMahan H, Ramage D, Richtárik P. Federated Optimization: Distributed Machine Learning for On-Device Intelligence. arXiv. 2016 Oct 8. URL: http://arxiv.org/abs/1610.02527 [accessed 2019-11-15]

22. Dean J, Corrado G, Monga R, Chen K, Devin M, Mao M, et al. Large Scale Distributed Deep Networks. Red Hook, NY: Curran Associates Inc; 2012 Dec Presented at: 25th International Conference on Neural Information Processing Systems; December 2012; Lake Tahoe, Nevada p. 1223-1231 URL: https://dl.acm.org/doi/10.5555/2999134.2999271

23. Sarwate AD, Chaudhuri K. Signal Processing and Machine Learning with Differential Privacy: Algorithms and challenges for continuous data. IEEE Signal Process Mag 2013 Sep 01;30(5):86-94 [FREE Full text] [doi: 10.1109/MSP.2013.2259911] [Medline: 24737929]

24. Ma C, Konečný J, Jaggi M, Smith V, Jordan MI, Richtárik P, et al. Distributed optimization with arbitrary local solvers. Optim Method Soft 2017 Feb 31;32(4):813-848. [doi: 10.1080/10556788.2016.1278445]

25. Shamir O, Srebro N, Zhang T. Communication-Efficient Distributed Optimization using an Approximate Newton-type Method. 2014 Jan Presented at: 31st International Conference on Machine Learning (ICML 2014); June 21-26, 2014; Beijing, China p. 1000-1008 URL: http://proceedings.mlr.press/v32/shamir14.pdf

26. Zhang Y, Xiao L. DiSCO: Distributed optimization for self-concordant empirical loss. 2015 Jun 1 Presented at: 32nd International Conference on Machine Learning (ICML 2015); July 6-11, 2015; Lille, France p. 362-370 URL: http:/ /proceedings.mlr.press/v37/zhangb15.pdf

27. Chilimbi T, Suzue Y, Apacible J, Kalyanaraman K. Project Adam: Building an Efficient and Scalable Deep Learning Training System. 2014 Presented at: 11th USENIX Symposium on Operating Systems Design and Implementation (OSDI 14); October 6-8, 2014; Broomfield, CO p. 571-582 URL: https://www.usenix.org/conference/osdi14/technical-sessions/ presentation/chilimbi

28. Konečný J, McMahan H, Yu F, Richtárik P, Suresh A, Bacon D. Federated Learning: Strategies for Improving Communication Efficiency. arXiv.: NIPS Workshop on Private Multi-Party Machine Learning (2016); 2017 Oct 30. URL: https://arxiv.org/ abs/1610.05492 [accessed 2019-11-15]

29. McMahan H, Moore E, Ramage D, Hampson S. Communication-Efficient Learning of Deep Networks from Decentralized Data. 2017 Presented at: 20th International Conference on Artificial Intelligence and Statistics (AISTATS) 2017; May 9-11, 2017; Fort Lauderdale, FL p. 1273-1282 URL: http://proceedings.mlr.press/v54/mcmahan17a/mcmahan17a.pdf

30. McMahan H, Moore E, Ramage D, Arcas B. Federated Learning of Deep Networks using Model Averaging. arXiv. 2016 Feb 17. URL: https://uk.arxiv.org/pdf/1602.05629v1.pdf [accessed 2019-11-15]

31. Abadi M, Chu A, Goodfellow I, McMahan H, Mironov I, Talwar K, et al. Deep Learning with Differential Privacy. 2016 Oct 24 Presented at: 2016 ACM SIGSAC Conference on Computer and Communications Security; October 24-28, 2016; Hofburg Palace, Vienna, Austria p. 308-318 URL: https://dl.acm.org/doi/10.1145/2976749.2978318 [doi: $10.1145 / 2976749.2978318]$

32. Geyer R, Klein T, Nabi M. Differentially Private Federated Learning: A Client Level Perspective. arXiv. 2018 May 1. URL: https://arxiv.org/abs/1712.07557 [accessed 2019-11-15]

33. Bonawitz K, Ivanov V, Kreuter B, Marcedone A, McMahan H, Patel S, et al. Practical Secure Aggregation for Privacy-Preserving Machine Learning. New York, NY: Association for Computing Machinery; 2017 Oct 30 Presented at: 
2017 ACM SIGSAC Conference on Computer and Communications Security; October 30-November 3, 2017; Dallas, TX p. 1175-1191 URL: https://dl.acm.org/doi/abs/10.1145/3133956.3133982 [doi: 10.1145/3133956.3133982]

34. Bonawitz K, Eichner H, Grieskamp W, Huba D, Ingerman A, Ivanov V, et al. Towards Federated Learning at Scale: System Design. 2019 Mar 22 Presented at: 2nd SysML Conference 2019; March 31-April 2, 2019; Palo Alto, CA URL: https:/ larxiv.org/abs/1902.01046

35. Yang T, Andrew G, Eichner H, Sun H, Li W, Kong N, et al. Applied Federated Learning: Improving Google Keyboard Query Suggestions. arXiv. 2018 Dec 7. URL: https://arxiv.org/abs/1812.02903 [accessed 2019-11-15]

36. Hard A, Rao K, Mathews R, Ramaswamy S, Beaufays F, Augenstein S, et al. Federated Learning for Mobile Keyboard Prediction. arXiv. 2019 Feb 28. URL: https://arxiv.org/abs/1811.03604 [accessed 2019-11-15]

37. Bagdasaryan E, Veit A, Hua Y, Estrin D, Shmatikov V. How To Backdoor Federated Learning. : PMLR; 2019 Aug 6 Presented at: 23rd International Conference on Artificial Intelligence and Statistics (AISTATS 2020); August 26-28, 2020; Online p. 2938-2948 URL: http://proceedings.mlr.press/v108/bagdasaryan20a.html

38. Yang T, Chen Y, Sze V. Designing Energy-Efficient Convolutional Neural Networks Using Energy-Aware Pruning. : IEEE; 2017 Nov 9 Presented at: 2017 IEEE Conference on Computer Vision and Pattern Recognition (CVPR); July 21-26, 2017; Honolulu, HI p. 6071-6079 URL: https://ieeexplore.ieee.org/document/8100126 [doi: 10.1109/CVPR.2017.643]

39. He Y, Zhang X, Sun J. Channel Pruning for Accelerating Very Deep Neural Networks. : IEEE; 2017 Dec 25 Presented at: 2017 IEEE International Conference on Computer Vision (ICCV); October 22-29, 2017; Venice, Italy p. 1398-1406 URL: https://ieeexplore.ieee.org/document/8237417 [doi: 10.1109/iccv.2017.155]

40. Han H, Qiao J. A Self-Organizing Fuzzy Neural Network Based on a Growing-and-Pruning Algorithm. IEEE Trans Fuzzy Syst 2010 Dec;18(6):1129-1143. [doi: 10.1109/TFUZZ.2010.2070841]

41. Srinivas S, Babu R. Data-free parameter pruning for Deep Neural Networks. : BMVC Press; 2015 Jul 22 Presented at: 26th British Machine Vision Conference (BMVC 2015); September 7-10, 2015; Swansea, UK URL: https://arxiv.org/abs/1507. $\underline{06149}$ [doi: $\underline{10.5244 / \text { c. } 29.31]}$

42. Hu H, Peng R. Network Trimming: A Data-Driven Neuron Pruning Approach towards Efficient Deep Architectures. arXiv. 2016 Jul 12. URL: https://arxiv.org/abs/1607.03250 [accessed 2019-11-15]

43. Dwork C, Roth A. The Algorithmic Foundations of Differential Privacy. FNT in Theoretical Computer Science 2014;9(3-4):211-407. [doi: 10.1561/0400000042]

44. Dwork C, McSherry F, Nissim K, Smith A. Calibrating Noise to Sensitivity in Private Data Analysis. In: Halevi S, Rabin T, editors. Theory of Cryptography. Berlin, Heidelberg: Springer; 2006:265-284.

45. Dwork C, Naor M, Pitassi T, Rothblum G. Differential privacy under continual observation. New York, NY: Association for Computing Machinery; 2010 Presented at: 42nd ACM symposium on Theory of computing; June 5-8, 2010; Cambridge, MA p. 715-724. [doi: 10.1145/1806689.1806787]

46. Dwork C, Rothblum G, Vadhan S. Boosting and Differential Privacy. : IEEE; 2010 Presented at: 2010 IEEE 51st Annual Symposium on Foundations of Computer Science; October 23-26, 2010; Las Vegas, NV p. 51-60 URL: https://ieeexplore. ieee.org/document/5670947 [doi: 10.1109/focs.2010.12]

47. Fredrikson M, Jha S, Ristenpart T. Model Inversion Attacks that Exploit Confidence Information and Basic Countermeasures. New York, NY: Association for Computing Machinery; 2015 Presented at: 22nd ACM SIGSAC Conference on Computer and Communications Security; October 12-16, 2015; Denver, CO p. 1322-1333 URL: https://dl.acm.org/doi/10.1145/ 2810103.2813677 [doi: $10.1145 / 2810103.2813677$ ]

48. Song S, Chaudhuri K, Sarwate A. Stochastic gradient descent with differentially private updates. : IEEE; 2013 Presented at: 2013 IEEE Global Conference on Signal and Information Processing; December 3-5, 2013; Austin, TX p. 245-248 URL: https://ieeexplore.ieee.org/document/6736861 [doi: 10.1109/globalsip.2013.6736861]

49. Bassily R, Smith A, Thakurta A. Private Empirical Risk Minimization: Efficient Algorithms and Tight Error Bounds. : IEEE; 2014 Presented at: 2014 IEEE 55th Annual Symposium on Foundations of Computer Science; October 18-21, 2014; Philadelphia, PA p. 464-473 URL: https://ieeexplore.ieee.org/document/6979031 [doi: 10.1109/focs.2014.56]

\section{Abbreviations}

APoZ: average percentage of zeros

AUC-PR: area under the precision-recall curve

AUC-ROC: area under the receiver operating characteristic curve

FN: false negative

FP: false positive

FPR: false positive rate

ReLU: rectified linear unit

SCBFL: stochastic channel-based federated learning

SCBFLwP: stochastic channel-based federated learning with pruning

SGD: stochastic gradient descent

TN: true negative 
TP: true positive

TPR: true positive rate

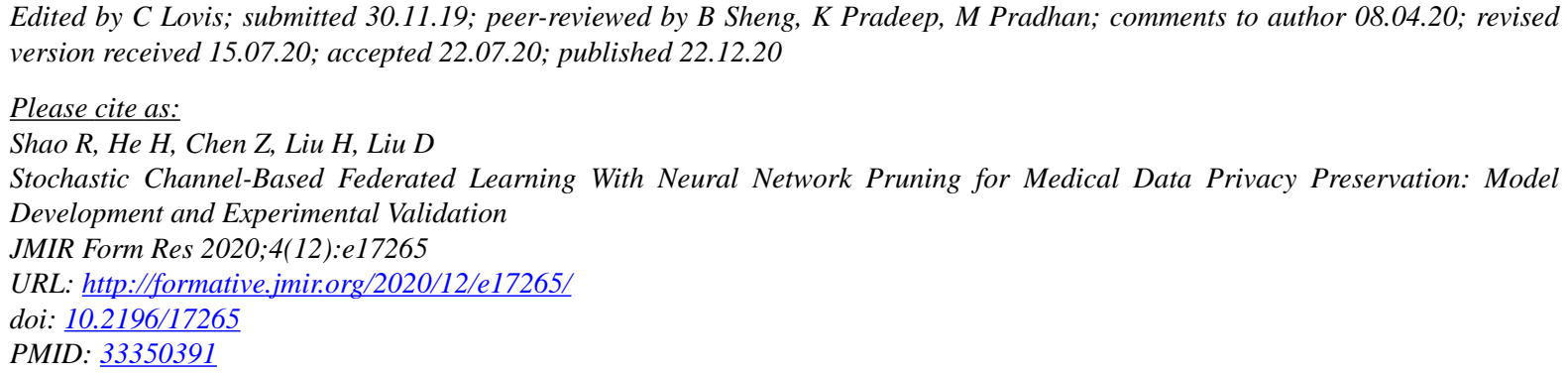

(CRulin Shao, Hongyu He, Ziwei Chen, Hui Liu, Dianbo Liu. Originally published in JMIR Formative Research (http://formative.jmir.org), 22.12.2020. This is an open-access article distributed under the terms of the Creative Commons Attribution License (https://creativecommons.org/licenses/by/4.0/), which permits unrestricted use, distribution, and reproduction in any medium, provided the original work, first published in JMIR Formative Research, is properly cited. The complete bibliographic information, a link to the original publication on http://formative.jmir.org, as well as this copyright and license information must be included. 